\title{
A simple one-step chemistry model for partially premixed hydrocarbon combustion
}

\author{
Eduardo Fernández-Tarrazo ${ }^{\mathrm{a}}$, Antonio L. Sánchez ${ }^{\mathrm{b}, *}$, Amable Liñán ${ }^{\mathrm{c}}$, \\ Forman A. Williams ${ }^{\mathrm{d}}$ \\ a Instituto Nacional de Técnica Aeroespacial, Madrid, Spain \\ b Area de Mecánica de Fluidos, Universidad Carlos III de Madrid, Leganés 28911, Spain \\ ${ }^{\mathrm{c}}$ ETSI Aeronáuticos, Pl. Cardenal Cisneros 3, Madrid 28040, Spain \\ ${ }^{\mathrm{d}}$ Department of Mechanical and Aerospace Engineering, University of California San Diego, La Jolla, CA 92093-0411, USA \\ Received 13 March 2006; received in revised form 28 July 2006; accepted 2 August 2006 \\ Available online 18 September 2006
}

\section{Introduction}

Despite the increase in computer power, there is interest in simple model chemistry descriptions that reproduce the main features of the combustion process accurately without excessive computer

\footnotetext{
* Corresponding author. Fax: +34916249430.

E-mail address: asanchez@ing.uc3m.es (A.L. Sánchez).
}

cost. These simple models are particularly suited to computations of turbulent flows, for which detailed chemistry descriptions are prohibitively expensive. For many purposes, one does not require the level of detailed information that can be extracted from a detailed chemistry computation, so that the lower computational cost of a simpler chemistry description is preferred. This type of approach is adopted, for instance, in two recent works, a DNS analysis of imperfectly premixed combustion [1] and a 
numerical description of diffusion-flame liftoff and blowoff [2].

Efforts to generate simple chemistry models trace back to the early work of Westbrook and Dryer [3], who considered in particular one-step irreversible Arrhenius kinetics, along with two-step kinetics with $\mathrm{CO}$ as dominant intermediate species. Their development was based on computations of laminar premixed flames, leading to a set of optimum chemical-kinetic parameters (activation energy, preexponential factor, and reaction orders of fuel and oxidizer) that best fitted the main features of premixed combustion.

This paper explores the suitability of one-step irreversible Arrhenius kinetics to the description of partially premixed hydrocarbon combustion. Combustion in nonuniform mixtures propagates along the stoichiometric surfaces in the form of triple flames, with lean and rich premixed branches and a trailing diffusion flame. The model chemistry to be developed therefore needs to describe accurately both premixed and nonpremixed combustion, including specific key aspects such as flame temperatures, laminar flame propagation velocities over the whole flammability range, and critical conditions for strain-induced extinction.

\section{The model chemistry}

The development considers a single irreversible reaction between the hydrocarbon and the oxygen of the air,

$\mathrm{C}_{n} \mathrm{H}_{m}+\left(n+\frac{m}{4}\right) \mathrm{O}_{2} \rightarrow n \mathrm{CO}_{2}+\frac{m}{2} \mathrm{H}_{2} \mathrm{O}+q$,

with a global rate of the form

$\omega=B e^{-T_{\mathrm{a}} / T} C_{\mathrm{C}_{n} \mathrm{H}_{m}} C_{\mathrm{O}_{2}}$,

where $C_{i}$ is the concentration of species $i$. Nonunity reaction orders, proposed for instance in [3], are known to add numerical stiffness to the computation [4], so that the present development assumes unity reaction orders for the fuel and oxidizer. With that assumption, three different parameters are left in the model, namely, the preexponential factor, $B$, the activation temperature, $T_{\mathrm{a}}$, and the amount of heat released per mole of fuel consumed, $q$. We start the model selection by considering this last parameter and the resulting adiabatic flame temperature, given in Fig. 1. Values of $B$ and $T_{\mathrm{a}}$ are selected next, based on results of computations of planar premixed flames, shown in Fig. 2, and the capability of the model for describing strained premixed flames and nonpremixed combustion is tested in Figs. 3 and 4, respectively, where results of counterflow flames are compared with detailed chemistry computations. All

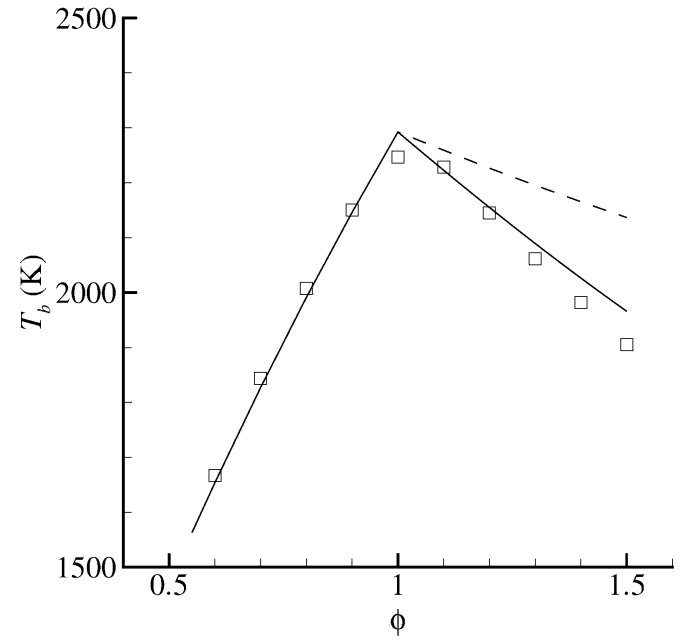

Fig. 1. The variation with equivalence ratio of the adiabatic flame temperature of methane-air combustion obtained from chemical equilibrium computations (symbols), with constant heat release $q=q_{\mathrm{o}}$ (dashed line), and with Eq. (5) (solid line).

computations correspond to methane-air combustion, although the selection procedure for the different model parameters and the accompanying discussion pertains to general hydrocarbons.

\section{Heat release}

With $\mathrm{CO}_{2}$ and $\mathrm{H}_{2} \mathrm{O}$ being the products of the hydrocarbon oxidation, the amount of heat released per mole of fuel consumed is

$q_{\mathrm{O}}=h_{\mathrm{C}_{n} \mathrm{H}_{m}}-n h_{\mathrm{CO}_{2}}-(m / 2) h_{\mathrm{H}_{2} \mathrm{O}}$,

where $h_{i}$ represents the enthalpy of formation per mol of species $i$, giving for instance $q_{\mathrm{o}}=802.4 \mathrm{~kJ} / \mathrm{mol}$ for methane. Fig. 1 compares the variation with equivalence ratio, $\phi$, of the adiabatic flame temperature $T_{\mathrm{b}}$ of methane-air combustion obtained with $q=q_{\text {o }}$ with that resulting from chemical equilibrium. The computation considers atmospheric pressure and initial temperature $T_{\mathrm{u}}=300 \mathrm{~K}$. As can be seen, the approximation $q=802.4 \mathrm{~kJ} / \mathrm{mol}$ is very accurate for $\phi<1$, but results in severe overpredictions of $T_{\mathrm{b}}$ for rich flames.

To understand the discrepancies found for $\phi>1$, one needs to consider the composition of the combustion products of hydrocarbon-air combustion. In the one-step model, the mixture at equilibrium is composed of $\mathrm{CO}_{2}$ and $\mathrm{H}_{2} \mathrm{O}$, together with the inert (typically the $\mathrm{N}_{2}$ of the air) and the excess reactant, i.e., $\mathrm{O}_{2}$ in lean mixtures and $\mathrm{C}_{n} \mathrm{H}_{m}$ in rich mixtures. An equilibrium calculation shows that this assumption is quite accurate for lean conditions, which explains the 
agreement found in Fig. 1 for $\phi<1$. However, in fuelrich combustion, almost all of the fuel is consumed to give $\mathrm{CO}_{2}$ and $\mathrm{H}_{2} \mathrm{O}$, along with significant amounts of $\mathrm{CO}$ and $\mathrm{H}_{2}$. Except near stoichiometric conditions, the presence of $\mathrm{O}_{2}$ at equilibrium is negligible, and so is that of hydrogen-oxygen radicals, which only appear in significant amounts in the combustion of preheated mixtures leading to burnt temperatures higher than $T_{\mathrm{b}} \simeq 2500 \mathrm{~K}$. Therefore, when considering the combustion energy balance for $\phi>1$, one can assume $\mathrm{CO}_{2}, \mathrm{H}_{2} \mathrm{O}$, CO, and $\mathrm{H}_{2}$ to be the main combustion products, thereby giving

$$
\begin{aligned}
q= & -(2 n+m / 2) h_{\mathrm{H}_{2} \mathrm{O}}+\phi\left[h_{\mathrm{C}_{n} \mathrm{H}_{m}}-n\left(h_{\mathrm{CO}_{2}}\right.\right. \\
& \left.\left.-2 h_{\mathrm{H}_{2} \mathrm{O}}\right)+N_{\mathrm{CO}}\left(h_{\mathrm{CO}_{2}}-h_{\mathrm{CO}}-h_{\mathrm{H}_{2} \mathrm{O}}\right)\right],
\end{aligned}
$$

where $N_{\mathrm{CO}}$ is the number of moles of carbon monoxide produced per mole of fuel consumed. One can simplify the above expression by noting that the factor multiplying $N_{\mathrm{CO}}$ is relatively small and can therefore be neglected in the first approximation. With this simplification, the resulting function $q(\phi)$ for the Arrhenius model becomes

$$
\left\{\begin{array}{l}
\phi \leqslant 1: q / q_{\mathrm{o}}=1, \\
\phi \geqslant 1: q / q_{\mathrm{o}}=1-\alpha(\phi-1),
\end{array}\right.
$$

where $\alpha=-\left[h_{\mathrm{C}_{n} \mathrm{H}_{m}}-n\left(h_{\mathrm{CO}_{2}}-2 h_{\mathrm{H}_{2} \mathrm{O}}\right)\right] / q_{\mathrm{o}}$ is a constant that changes by a small amount for different hydrocarbons. For instance, $\alpha=0.21$ for $\mathrm{CH}_{4}$ and $\alpha=0.18$ for $\mathrm{C}_{2} \mathrm{H}_{6}, \mathrm{C}_{3} \mathrm{H}_{8}, \mathrm{C}_{4} \mathrm{H}_{10}$, and $\mathrm{C}_{7} \mathrm{H}_{16}$. As seen in Fig. 1, the adiabatic flame temperature obtained with this variable $q(\phi)$ is reasonably accurate. Small departures are found at stoichiometric conditions, due to the presence of a nonnegligible amount of $\mathrm{O}_{2}$ in the combustion products, and also for very rich flames, when the last term in (4), neglected when writing (5), leads to slight overpredictions of $T_{\mathrm{b}}$.

\section{Activation temperature}

Two more parameters need to be selected to complete the definition of the model: the preexponential factor $B$ and the activation temperature $T_{\mathrm{a}}$. In the selection, which is based on computations of propagation velocities $v_{1}$ of planar premixed flames with detailed chemistry, one should bear in mind that for one-step Arrhenius kinetics with high activation temperature the value of $T_{\mathrm{a}}$ determines the sensitivity of the flame response to changes in $T_{\mathrm{b}}$ according to $v_{1}^{2} \propto \exp \left(-T_{\mathrm{a}} / T_{\mathrm{b}}\right)$. The corresponding dependence for the case of hydrocarbon-air flames has been identified in previous rate-ratio asymptotic analyses based on reduced chemistry descriptions [5-8]. A two-layer flame structure consisting of a very thin fuel consumption layer and a much thicker $\mathrm{CO}$ oxidation layer is seen to apply under stoichiometric [5] and lean conditions [6,7], and also for moderately rich flames [8]. Under these conditions, the flame velocity dependence on the flame temperature is given by $v_{1}^{2} \propto\left(T_{\mathrm{b}}-T^{0}\right)^{\alpha} /\left(T_{\mathrm{b}}-T_{\mathrm{u}}\right)^{\alpha}$, where $T^{0}$ represents the temperature at the fuel-consumption layer. Values of the numerical exponent $\alpha$ are given in [6] for different hydrocarbons, along with numerical fits of $T^{0}$ as a function of $\phi \leqslant 1$ and pressure.

By equating the expression for the derivative of $\ln \left(v_{1}^{2}\right)$ with respect to $\ln \left(T_{\mathrm{b}}\right)$ obtained in the reducedchemistry analyses with that obtained with the onestep Arrhenius chemistry in the limit of large activation temperatures, one arrives at the simple equation [9]

$T_{\mathrm{a}}=\frac{\alpha T_{\mathrm{b}}^{2}\left(T^{0}-T_{\mathrm{u}}\right)}{\left(T_{\mathrm{b}}-T^{0}\right)\left(T_{\mathrm{b}}-T_{\mathrm{u}}\right)}$,

which can be used to evaluate $T_{\mathrm{a}}$ for different values of $T_{\mathrm{b}}, T_{\mathrm{u}}$, and $T^{0}$. For instance, for methane-air combustion ( $\alpha=4$ ), the burnt temperature corresponding to $T_{\mathrm{u}}=300 \mathrm{~K}$ is given in Fig. 1 , while the value of $T^{0}$ can be taken from the previous studies [6-8], giving for instance $T^{0} \simeq(1200,1300,1500,1750) \mathrm{K}$ for $\phi=(0.5,0.7,1.0,1.2)$ and $p=1 \mathrm{~atm}$. It turns out that the resulting value of $T_{\mathbf{a}}$ is rather independent of the composition for stoichiometric and moderately lean flames, i.e., $T_{\mathrm{a}}=15,870 \mathrm{~K}$ for $\phi=1$ and $T_{\mathrm{a}}=16,060 \mathrm{~K}$ for $\phi=0.7$, but becomes larger outside this range, i.e., $T_{\mathrm{a}}=22,500 \mathrm{~K}$ for $\phi=0.5$ and $T_{\mathrm{a}}=48,300 \mathrm{~K}$ for $\phi=1.2$. This finding justifies the previous model introduced in [1], where a single pair of values $\left(T_{\mathrm{a}}, B\right)$ was used to represent premixed combustion for compositions ranging from moderately lean to stoichiometric mixtures, whereas variations of $T_{\mathrm{a}}$ with $\phi$ were introduced for very lean flames and also for rich flames.

Given the functional dependences $T_{\mathrm{b}}\left(T_{\mathrm{u}}, \phi\right)$ and $T^{0}(p, \phi)$, Eq. (6) provides a means for determining $T_{\mathrm{a}}$ for given values of $\phi, p$, and $T_{\mathrm{u}}$. The procedure can be implemented in numerical computations of nonuniform mixtures, for which the use of a passive scalar enables the computation of the local values of $\phi$ and $T_{\mathrm{u}}$, as seen below. The main limitation of this approach pertains to rich mixtures, for which evaluation of (6) with the inner temperature deduced from the reduced chemistry analysis [8] is seen to give values of $T_{\mathrm{a}}$ that are much too large for $\phi \geqslant 1.1$ and become infinite as $T^{0}$ approaches $T_{\mathrm{b}}$, which occurs at $\phi=1.36$ for atmospheric methane-air combustion [8]. Equation (6) is no longer valid for $\phi>1.36$, when the two-layer flame structure is replaced by a single reaction layer, a problem analyzed in [10]. In principle, the dependence of $v_{1}^{2}$ on $T_{\mathrm{b}}$ could be extracted from the asymptotic results [10], leading to an alternative to Eq. (6) for rich mixtures. Unfortunately, the 
analysis of these fuel-rich flames yields expressions for $v_{1}$ with errors that exceed $100 \%$ when compared with the detailed chemistry computations (see Fig. 7 in [10]). Therefore, the development, based on [10], of an alternative to Eq. (6) for fuel-rich mixtures is abandoned, because the anticipated inaccuracies in $T_{\mathrm{a}}$ would be too large.

In view of these limitations, the development below employs a simpler approach, involving the use of an activation temperature $T_{\mathrm{a}}(\phi)$ that remains constant for stoichiometric and moderately lean flames but increases for very lean and for rich flames, as suggested by the previous evaluations of (6). The chemistry model using this function $T_{\mathrm{a}}(\phi)$, adjusted to reproduce $v_{1}(\phi)$ for a methane-air mixture with $T_{\mathrm{u}}=300 \mathrm{~K}$ and $p=1 \mathrm{~atm}$, is seen to reproduce with sufficient accuracy different aspects of atmospheric premixed and nonpremixed combustion, motivating further use of the one-step model. The application to fuels different from methane, or at nonatmospheric pressures, or at initial temperatures different from $T_{\mathrm{u}}=300 \mathrm{~K}$ will clearly require the computation of a different $T_{\mathrm{a}}(\phi)$. As suggested by a referee, future efforts may alternatively consider the use of (6) to determine the local value of the activation temperature, with the function $T^{0}(p, \phi)$ taken from [6] for $\phi \lesssim 1$, whereas for sufficiently rich flames the model should employ heuristic expressions for $T^{0}(p, \phi)$, adjusted for instance to reproduce accurately flame propagation velocities for $\phi$ somewhat greater than unity.

\section{Burning velocity}

Guided by the previous estimates obtained from (6) at $\phi=0.7$ and $\phi=1.0$, we start by considering a constant value of the activation temperature,

$T_{\mathrm{ao}}=15,900 \mathrm{~K}$,

and computing the accompanying preexponential factor,

$B=6.9 \times 10^{14} \mathrm{~cm}^{3} /(\operatorname{mol~s})$,

for which the resulting stoichiometric flame propagation velocity equals that obtained experimentally. This pair of constant parameters is then used to compute $v_{1}$ for different values of $\phi$, giving the results shown in Fig. 2. In a first series of computations, a constant heat of reaction $q=q_{\mathrm{o}}$ is employed, giving values of $v_{1}$ that agree for $\phi$ in the intermediate range $0.6 \leqslant \phi \leqslant 1.0$ with values measured experimentally $[11,12]$. Departures are observed outside this range, with the larger inaccuracies found for rich conditions, when the model chemistry erroneously predicts an increasing value of $v_{1}$ with $\phi$, a behavior previously

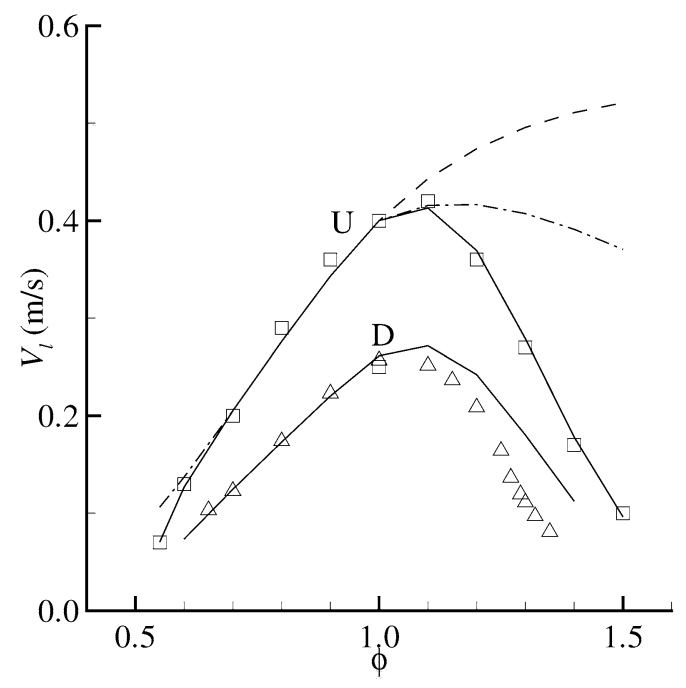

Fig. 2. The variation with equivalence ratio of the flame propagation velocity as obtained from experiments $[11,12]$ (squares), from detailed chemistry computations using the GRI mechanism [13] (triangles), and from one-step chemistry computations with $T_{\mathrm{a}}=T_{\mathrm{ao}}$ and $q=q_{\mathrm{o}}$ (dashed line), with $T_{\mathrm{a}}=T_{\mathrm{ao}}$ and $q=q(\phi)$ (dot-dashed line), and with $T_{\mathrm{a}}(\phi)$ and $q(\phi)$ (solid lines). The $\mathrm{U}$ indicates results for a methane-air mixture while the $\mathrm{D}$ indicates results for a methane-oxygen-nitrogen mixture with relative nitrogen-oxygen volume fraction $\mathrm{N}_{2} /\left(\mathrm{N}_{2}+\mathrm{O}_{2}\right)=0.82$.

noted in [3]. The selection $T_{\mathrm{a}}=15,900 \mathrm{~K}$ obtained from the reduced kinetic result (6) seems to be optimal, in that either larger or smaller values of the activation temperature worsen the response for moderately lean flames.

As can be seen in the figure, better accuracy is obtained when the variable heat release $q(\phi)$ given in (5) is used to compute rich flames, although the modification does not suffice to give accurate results for $\phi \gtrsim 1$.1. Further model improvements to mimic the changes occurring in the underlying chemistry require consideration of changes in the activation temperature. Keeping the value $B$ given in Eq. (8), for each value of $\phi$, the value of $T_{\mathrm{a}}$ that provides the exact value of $v_{\mathrm{l}}$ was computed. The resulting function $T_{\mathrm{a}}(\phi)$ is well approximated by the piecewise expression

$$
\begin{cases}\phi \leqslant 0.64: & T_{\mathrm{a}} / T_{\mathrm{ao}} \\ & =1+8.250(\phi-0.64)^{2}, \\ 0.64 \leqslant \phi \leqslant 1.07: & T_{\mathrm{a}} / T_{\mathrm{ao}}=1, \\ \phi \geqslant 1.07: & T_{\mathrm{a}} / T_{\mathrm{ao}} \\ & =1+1.443(\phi-1.07)^{2},\end{cases}
$$

which results in accurate premixed flame velocities as shown in Fig. 2. 


\section{Effects of dilution and strain on premixed flames}

It is of interest to test whether the model, whose parameters were adjusted to mimic premixed flame velocities in unstrained fuel-air mixtures, remains accurate in the presence of dilution or strain. The former effect is addressed by considering $\mathrm{N}_{2}$ as diluent, leading to mixtures where the nitrogen-oxygen relative composition exceeds that of air, $\mathrm{N}_{2} /\left(\mathrm{N}_{2}+\right.$ $\left.\mathrm{O}_{2}\right)=0.79$. The results are quite satisfactory, as can be seen in Fig. 2, where the variation of $v_{1}$ with $\phi$ computed with the one-step model for a methaneoxygen-nitrogen mixture with a relative volume fraction $\mathrm{N}_{2} /\left(\mathrm{N}_{2}+\mathrm{O}_{2}\right)=0.82$ is compared with the value determined experimentally in [12] for a stoichiometric mixture and with results of detailed chemistry computations for nonstoichiometric mixtures.

To evaluate the effect of strain on atmospheric methane-air premixed flames, the fresh-to-fresh planar counterflow configuration considered in [14] was computed for different dilutions and strain rates up to extinction conditions. Critical values of the resulting strain rate at extinction, $a_{\mathrm{c}}$, are shown in Fig. 3 for different equivalence ratios. Detailed chemistry results include computations performed with the GRI mechanism [13] (triangles) and those reported in [14] (squares). As can be seen, the one-step model with variable $T_{\mathrm{a}}(\phi)$ and $q(\phi)$ gives reasonable agreement for lean and stoichiometric conditions, but overpre-

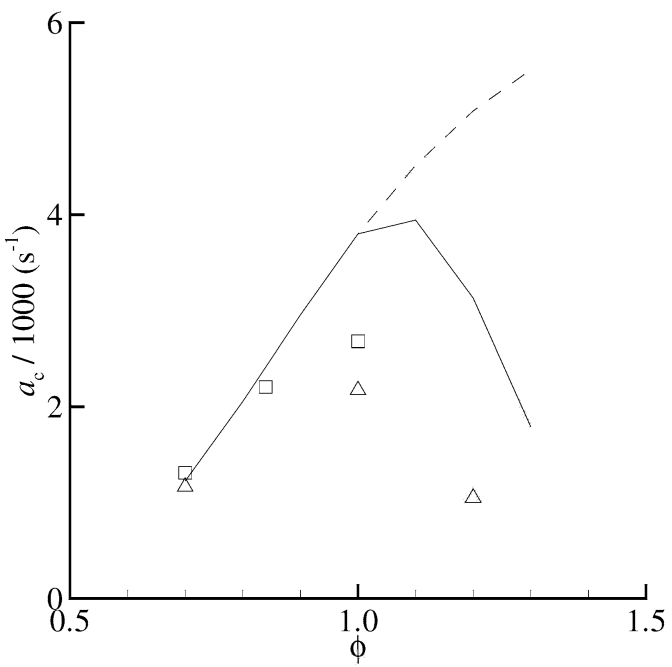

Fig. 3. The variation with equivalence ratio of the critical strain rate at extinction for a methane-air premixed flame in a planar fresh-to-fresh counterflow configuration as calculated with detailed chemistry (triangles, GRI mechanism, [13]; squares, [14]) and with the one-step model with $T_{\mathrm{a}}=T_{\mathrm{ao}}$ and $q=q_{\mathrm{o}}$ (dashed line) and with $T_{\mathrm{a}}(\phi)$ and $q(\phi)$ (solid lines). dicts the value of $a_{\mathrm{c}}$ significantly for rich flames. This overprediction is even more pronounced when constant values of $T_{\mathrm{a}}=T_{\mathrm{ao}}$ and $q=q_{\mathrm{o}}$ are employed in the model, yielding a value of $a_{\mathrm{c}}$ that erroneously increases with $\phi$ for $\phi>1$. This result is not surprising in view of the prediction of $v_{1}$ for $\phi>1$ shown in Fig. 2, further demonstrating the need for variable $T_{\mathrm{a}}(\phi)$ and $q(\phi)$ under fuel-rich conditions.

It is worth pointing out that, despite the reasonable agreement shown in Fig. 3 for $\phi \lesssim 1$, the computations of strained premixed flames revealed some predictive limitations of the one-step Arrhenius kinetics. For instance, when the model was used to compute adiabatic fresh-to-burnt counterflow configurations, the burning rate was seen to continuously decrease with increasing strain rate without ever achieving abrupt extinction, which is, however, captured in detailed chemistry computations [14]. Extinction in this case is a chemical-kinetic effect resulting from radical loss, which cannot be reproduced in the framework of our simple temperature dependent kinetics.

\section{Nonuniform mixtures}

The model is applicable to systems where the fuel and the oxidizer, typically the oxygen of the air, are supplied through different feed streams that partially premix before combustion occurs, leading to a nonuniform distribution of $\phi$ upstream from the reaction zone. To enable the numerical implementation of the model in such systems, one needs to relate the local equivalence ratio $\phi$, appearing in (5) and (9), to a variable that is conserved across the premixed flame. With unity Lewis numbers, one can use the mixture fraction variable $Z$, defined to be unity in the fuel stream and zero in the oxidizer stream. The equation $[1,2]$

$\phi=\frac{32(n+m / 4)}{12 n+m} \frac{Y_{\mathrm{C}_{n} \mathrm{H}_{m}, \mathrm{~F}}}{Y_{\mathrm{O}_{2}, \mathrm{~A}}} \frac{Z}{1-Z}$,

where $Y_{\mathrm{C}_{n} \mathrm{H}_{m}}$, F and $Y_{\mathrm{O}_{2}, \mathrm{~A}}=0.232$ denote the values of the fuel and oxygen mass fractions in their feed streams, can be used in (5) and (9) to determine the values of $T_{\mathrm{a}}$ and $q$ corresponding to the local conditions of mixing. The same procedure applies also to the determination of the local values of the model parameters in nonpremixed combustion. Also note that, in configurations where the fuel-stream temperature $T_{\mathrm{F}}$ differs from the oxidizer-stream temperature $T_{\mathrm{O}}$, the local unburnt temperature is determined from $T_{\mathrm{u}}=T_{\mathrm{O}}+\left(T_{\mathrm{F}}-T_{\mathrm{O}}\right) Z$, to be employed when determining the value of $T_{\mathrm{a}}$ from Eq. (6).

No conserved scalar can be defined when the fuel has a nonunity Lewis number. The mixture fraction 
used in (10) changes due to differential diffusion effects as one moves across the premixed flame, although the value of $Z$ that exists upstream in the fresh mixture is recovered as one approaches equilibrium downstream. Since the reaction zone tends to be located near the high-temperature equilibrium side of the flame, the error introduced when using $Z$ to determine the local values of the model parameters is not too large, thereby justifying the use of (10) also for fuels different from methane $[1,2]$.

\section{Nonpremixed flames}

Computations of planar methane-air counterflow flames were used to test the model under nonpremixed conditions, with (10) being employed to calculate the local values of the model parameters. Fig. 4 compares the variation with strain rate $a$ of the resulting peak temperature obtained with the model chemistry with results of previous detailed chemistry computations $[15,16]$. As can be seen, with constant values of the parameters, the model overpredicts both the temperature and the critical strain rate at extinction, $a_{\mathrm{c}}$, which is about a factor of 5 larger than that obtained with detailed chemistry. Much better agreement is obtained when the variable heat release (5) is incorporated in the one-step computation (the dot-dashed line in the figure), giving a prediction of strain rate at extinction that approaches that obtained with detailed chemistry. As can be observed, the performance of the one-step kinetics is even more satisfactory when the variation of $q(\phi)$ and $T_{\mathrm{a}}(\phi)$ is simultaneously considered in the computation.

One of the most noticeable limitations of one-step Arrhenius kinetics with constant activation temperature concerns the prediction of reactant leakage in nonpremixed combustion. It is known that in diffusion flames the oxygen tends to leak across the flame in increasing amounts as extinction is approached [17]. This is a chemical-kinetic effect resulting from the depletion of radicals by rapid fuel attack in the thin fuel-consumption layer. In the absence of radicals, the oxygen cannot be consumed and therefore penetrates to the fuel side in significant amounts. However, onestep Arrhenius kinetics with constant activation temperature tends to predict the opposite behavior; fuel, rather than oxygen, is seen to leak across the flame in undiluted diffusion flames near extinction. This flaw of the simple one-step chemistry is remedied with the present model: by considering an increasing activation temperature on the fuel side of the flame, Eq. (9) mimics appropriately the depletion of radicals by fuel attack, and allows the model to predict the correct reactant leakage behavior near extinction. This is illustrated in the inset of Fig. 4, where we show reactant and temperature profiles across the flame. As can be seen, the results of the model at extinction give significant oxygen leakage, in agreement with detailedchemistry computations $[15,16]$. It should be noted that oxygen leakage is not present at extinction in the calculations with $q(\phi)$ and constant $T_{\mathrm{a}}=T_{\mathrm{ao}}$ (the dot-dashed line in Fig. 4), indicating that consideration of variable activation temperatures is necessary to capture this chemical-kinetic effect.

\section{Concluding comments}

We have seen how effects of partial fuel oxidation and of reduced oxidation rate, occurring mainly in fuel-rich environments, can be successfully incorporated into a simple one-step Arrhenius model by consideration of variable values of the heat of reaction and of the activation temperature, respectively, overcoming modeling limitations previously noted. In particular, the model proposed describes with sufficient accuracy flame temperatures, premixed burning velocities, and near-extinction diffusion flames, thereby enabling numerical computations of premixed, nonpremixed, and partially premixed combustion.

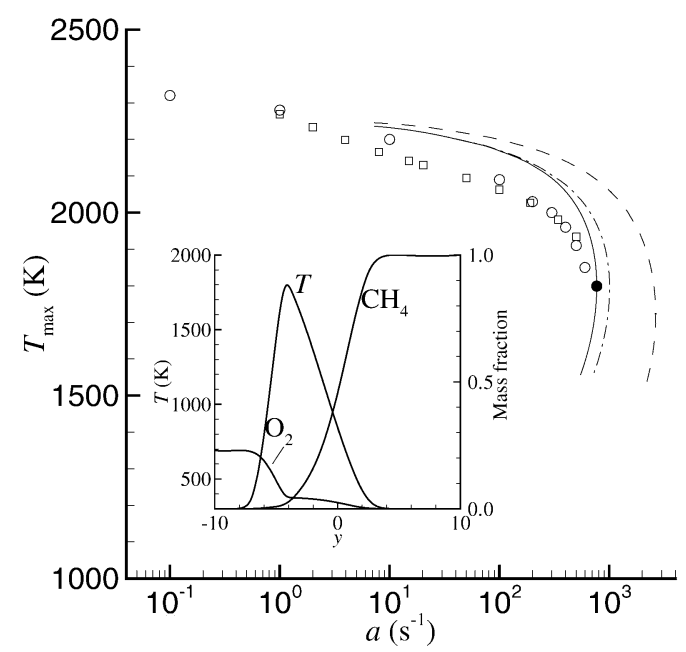

Fig. 4. The variation with strain rate of the peak temperature in a methane-air planar diffusion flame with feed temperatures $T_{\mathrm{u}}=300 \mathrm{~K}$ and pressure $p=1 \mathrm{~atm}$ as calculated with detailed chemistry (squares [15] and circles [16]), and with the one-step model (lines) with $T_{\mathrm{a}}=T_{\mathrm{ao}}$ and $q=q_{\mathrm{o}}$ (dashed line), with $T_{\mathrm{a}}=T_{\mathrm{ao}}$ and $q=q(\phi)$ (dot-dashed line) and with $T_{\mathrm{a}}(\phi)$ and $q(\phi)$ (solid lines). The inset shows temperature and reactant profiles obtained with the model for the near-extinction flame indicated by the black dot, with $y$ representing the distance to the stagnation plane scaled with $\left(D_{\mathrm{T}} / a\right)^{1 / 2}$, where $D_{\mathrm{T}}$ is the thermal diffusivity on the air side. 


\section{Acknowledgments}

The help of Mr. Pablo Martínez-Legazpi with numerical computations is gratefully acknowledged. This collaborative research was supported by the Spanish MEC under Projects ENE2005-08580-C02 and ENE2005-09190-C04-01, by the Comunidad de Madrid under Project S-505/ENE/0229, and by the National Science Foundation through Grant CTS 0129562 .

\section{References}

[1] D. Garrido-López, S. Sarkar, Proc. Combust. Inst. 30 (2005) 621-628.

[2] E. Fernández-Tarrazo, M. Vera, A. Liñán, Combust. Flame 144 (2006) 261-276.

[3] C. Westbrook, F. Dryer, Combust. Sci. Technol. 27 (1981) $31-43$.

[4] T. Poinsot, D. Veynante, Theoretical and Numerical Combustion, Edwards, Philadelphia, PA, 2001, p. 54.

[5] N. Peters, F.A. Williams, Combust. Flame 68 (1987) 185-207.

[6] J. Göttgens, F. Mauss, N. Peters, Proc. Combust. Inst.
24 (1992) 129-135.

[7] K. Seshadri, J. Göttgens, in: M.D. Smooke (Ed.), Reduced Kinetic Mechanisms and Asymptotic Approximations for Methane-Air Flames, in: Lectures Notes in Physics, vol. 384, Springer-Verlag, Berlin, 1991, p. 111.

[8] K. Seshadri, X.S. Bai, H. Pitsch, N. Peters, Combust. Flame 113 (1998) 589-602.

[9] A. Liñán, F.A. Williams, Fundamental Aspects of Combustion, Oxford Univ. Press, New York, 1993, p. 53.

[10] K. Seshadri, X.S. Bai, H. Pitsch, Combust. Flame 127 (2002) 2265-2277.

[11] F.N. Egolfopoulos, D.L. Zhu, C.K. Law, Proc. Combust. Inst. 23 (1990) 471-478.

[12] F.N. Egolfopoulos, C.K. Law, Combust. Flame 80 (1990) 7-16.

[13] M. Frenklach, T. Bowman, G. Smith, B. Gardiner, GRI-Mech 3.0 (30-7-99). Available at http://www.me. berkeley.edu/gri-mech.

[14] B. Rogg, Combust. Flame 73 (1988) 45-65.

[15] J. Hewson, M. Bollig, Proc. Combust. Inst. 26 (1996) 2171-2179.

[16] M. Bollig, A. Liñán, A.L. Sánchez, F.A. Williams, Proc. Combust. Inst. 27 (1998) 595-603.

[17] F.A. Williams, Prog. Energy Combust. Sci. 26 (2000) $657-682$. 